\title{
Simultaneous Information and Energy Transmission in Gaussian Interference Channels with Feedback
}

\author{
Nizar Khalfet and Samir M. Perlaza
}

\begin{abstract}
In this paper, the fundamental limits of simultaneous information and energy transmission in the two-user Gaussian interference channel with feedback are fully characterized. More specifically, an achievable and converse region in terms of information and energy transmission rates (in bits per channel use and energy-units per channel use, respectively) are presented. The achievable region is obtained using a combination of rate splitting, common randomness, superposition coding, powersplitting, and block Markov decoding. Finally, the converse region is obtained using some of the existing outer bounds on the information transmission rates, as well as a new outer bound on the energy transmission rate.
\end{abstract}

\section{INTRODUCTION}

Energy is a central resource in the process of data transmission and in some cases, it is a costly and rare resource. For instance, infrastructure-based communications are often powered by the electrical grid and often reinforced with sophisticated energy storage systems to increase reliability and optimize the use of alternative energy sources, e.g, solar panels, wind mills, etc. In this case, these networks can be thought as networks powered by an infinite-capacity battery with very low probability of power-shortage. Alternatively, mobile devices and some office, industrial and medical instruments are exclusively powered by batteries, which immediately suggests that infrastructure-less networks are more vulnerable to power shortages than infrastructure-based networks. When the energy-capacity of the battery is small, battery recharging and/or battery replacement must be regularly implemented. This observation highlights two relevant questions in this type of networks: (a) How to implement the battery recharging process to minimize the probability of energy outage?; and (b) How to efficiently burn the energy from the batteries to guarantee reliable communication?. This paper focuses on the first question assuming that energy can be harvested from radio frequency $(\mathrm{RF})$ signals sent by transmitters that are concerned with this task. More specifically, the focus is on the analysis of coding schemes for simultaneously transmitting information to a receiver and energy to an energy harvester that must guarantee a minimum energy rate, e.g., for battery recharging. The second question is tackled in [1], [2], [3], [4], [5] and

Nizar Khalfet and Samir M. Perlaza are with the Laboratoire CITI (a joint laboratory between the Université de Lyon, INSA de Lyon, and INRIA). 6 Avenue des Arts, F-69621, Villeurbanne, France. (\{nizar.khalfet, samir.perlaza\}@inria.fr).

Samir M. Perlaza is also with the Department of Electrical Engineering at Princeton University, Princeton, NJ.

This research was supported in part by the European Commission under Marie Skłodowska-Curie Individual Fellowship No. 659316 and Euro-Mediterranean Cooperation ERA-NET project COM-MED. references therein. Both questions are jointly studied in a specific configuration of the relay channel in [6].

The problem of wireless energy transmission traces back to Tesla in the 20-th century [7]. However, the problem of simultaneous information and energy transmission (SIET) has been formalized recently in the point-to-point channel [8], [9], [10]. The case of two transmitters, one receiver and one noncolocated energy harvester $(\mathrm{EH})$ has been studied in [11], and [12]. A comprehensive review of existing results in SIET in different multi-user channels is presented in [13].

This paper focuses on the case of the two-user Gaussian interference channel (G-IC) with perfect output feedback. The case without feedback has been studied in [14]. More specifically, this paper studies the case of two point-to-point channels subject to mutual interference with a non-colocated EH. The objective of both transmitters is to simultaneously transmit information to their corresponding intended receivers and jointly guarantee a minimum energy rate at the $\mathrm{EH}$. The main result is twofold. First, a set of achievable information and energy transmission rates is presented. That is, a subset of the information-energy capacity region [12]. Second, a set that contains the information capacity region. These two sets are shown to be a constant gap approximation of the set of triplets made of both information rates and the ratio between the energy rate and the maximum feasible energy rate. In this case, the gap is shown to be one bit in the information dimensions and one-half in the energy dimension. Note that the energy dimension does not have a unit as it is the ratio of two energy rates.

\section{Gaussian InTERfEREnCE Channel With EnERGy HARVESTER}

Consider the G-IC with a non-colocated energy harvester with feedback depicted in Fig. 1. Transmitter $i$, with $i \in$ $\{1,2\}$, aims to execute two tasks: $(a)$ an information transmission task and $(b)$ an energy transmission task.

\section{A. Information Transmission Task}

From the information transmission standpoint, the goal of transmitter $i$ is to convey an independent message index $W_{i} \in \mathcal{W}_{i}$ to receiver $i$ using $N$ channel input symbols $X_{i, 1}, X_{i, 2}, \ldots, X_{i, N}$. The channel coefficient from transmitter $k$ to receiver $i$, with $k \in\{1,2\}$, is denoted by $h_{i k} \in \mathbb{R}_{+}$. At receiver $i$, during channel use $n$, input symbol $X_{i, n}$ is observed at receiver $i$ subject to the interference produced by the symbol $X_{j, n}$ sent by transmitter $j$, with $j \in\{1,2\} \backslash\{i\}$, and a real additive Gaussian noise $Z_{i, n}$ with zero mean and variance $\sigma_{i}^{2}$. 
Hence, the channel output at receiver $i$ during channel use $n$, denoted by $Y_{i, n}$, is

$$
Y_{i, n}=h_{i i} X_{i, n}+h_{i j} X_{j, n}+Z_{i, n} .
$$

At each channel use $n$, the symbol $X_{i, n}$ sent by transmitter $i$ depends on the message index $W_{i}$, a randomly generated index $\Omega \in \mathbb{N}$, and all previous channel-outputs $Y_{i, 1}, Y_{i, 2}, \ldots, Y_{i, n-d}$, with $d \in \mathbb{N}$ the feedback delay. In the following, it is assumed that $d=1$ channel use, without any loss of generality. The first channel input symbol $X_{i, 1}$ depends only on the message index $W_{i}$ and $\Omega \in \mathbb{N}$. More specifically, $f_{i, 1}^{(N)}: \mathcal{W}_{i} \times \mathbb{N} \rightarrow \mathbb{R}$ and for all $n \in\{2,3, \ldots, N\}, f_{i, n}^{(N)}: \mathcal{W}_{i} \times \mathbb{N} \times \mathbb{R}^{n-1} \rightarrow \mathbb{R}$ are the encoding functions such that:

$$
X_{i, 1}=f_{i, 1}^{(N)}\left(W_{i}, \Omega\right)
$$

and for all $n \in\{2, \ldots, N\}$,

$$
X_{i, n}=f_{i, n}^{(N)}\left(W_{i}, \Omega, Y_{1}, \ldots, Y_{n-1}\right) .
$$

The random index $\Omega$ is assumed to be independent of both $W_{1}$ and $W_{2}$ and known by all transmitters and receivers. Channel input symbols $X_{i, 1}, X_{i, 2}, \ldots, X_{i, N}$ are subject to an average power constraint of the form

$$
\frac{1}{N} \sum_{n=1}^{N} \mathrm{E}\left[X_{i, n}^{2}\right] \leq P_{i},
$$

where $P_{i}$ denotes the average transmit power of transmitter $i$ in energy units per channel use. The expectation in (4) is taken with respect to $W_{i}, \Omega$, and the previous channel outputs $Y_{1}, \ldots, Y_{n-1}$. Note that $W_{i}$ and $\Omega$ are assumed to be uniformly distributed over their corresponding sets.

Let $T \in \mathbb{N}$ be fixed and assume that during a given communication, $T$ blocks of $N$ channel uses are transmitted. The decoder of receiver $i$ observes the channel outputs $Y_{i, 1}, Y_{i, 2}, \ldots, Y_{i, N T}$ and uses a decoding function

$$
\phi_{i}: \mathbb{N} \times \mathbb{R}^{N T} \rightarrow\left\{1,2, \ldots, 2^{R_{i}}\right\}^{\top},
$$

to get an estimate of the message indices:

$$
\left(\widehat{W}_{i}^{(1)}, \widehat{W}_{i}^{(2)}, \ldots, \widehat{W}_{i}^{(T)}\right)=\phi_{i}\left(\Omega, Y_{i, 1}, Y_{i, 2}, \ldots, Y_{i, N T}\right),
$$

where $\widehat{W}_{i}^{(t)}$ is an estimate of the message index sent during block $t \in\{1,2, \ldots, T\}$. The decoding error probability during block $t$ of a codebook of block-length $N$, denoted by $P_{\mathrm{e}}^{(t)}(N)$, is given by

$$
\begin{aligned}
& P_{\mathrm{e}}^{(t)}(N) \\
& =\max \left(\operatorname{Pr}\left[{\widehat{W_{1}}}^{(t)} \neq W_{1}^{(t)}\right], \operatorname{Pr}\left[{\widehat{W_{2}}}^{(t)} \neq W_{2}^{(t)}\right]\right) .
\end{aligned}
$$

The signal to noise ratio (SNR) at receiver $i$ is denoted by

$$
\mathrm{SNR}_{i}=\frac{\left|h_{i i}\right|^{2} P_{i}}{\sigma_{i}^{2}}
$$

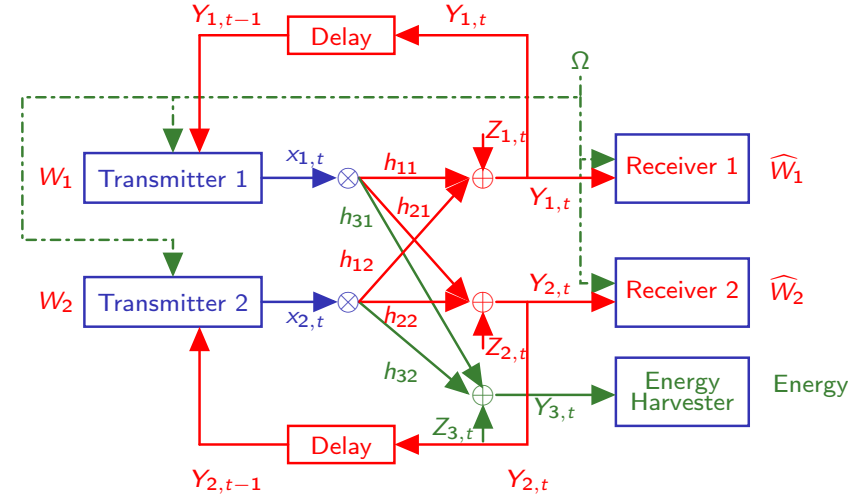

Fig. 1. Two-user Gaussian interference channel with a non-collocated energy harvester.

The interference to noise ratio (INR) at receiver $i$ is denoted by

$$
\mathrm{INR}_{i}=\frac{\left|h_{i j}\right|^{2} P_{j}}{\sigma_{i}^{2}}, \text { with } j \neq i
$$

\section{B. Energy Transmission Task}

Let $h_{3 i} \in \mathbb{R}_{+}$be the channel coefficient from transmitter $i$ to the energy harvester (EH). The symbols sent by the transmitters are observed by the EH subject to an additive Gaussian noise $Z_{3, n}$ with zero mean and variance $\sigma_{3}^{2}$. More specifically, the channel output at the EH, denoted by $Y_{3, n}$, is

$$
Y_{3, n}=h_{31} X_{1, n}+h_{32} X_{2, n}+Z_{3, n} .
$$

From the energy transmission standpoint, the goal of both transmitters is to jointly guarantee an average energy rate at the EH. Let $b \geqslant 0$ denote the minimum average energy rate that must be guaranteed at the input of the EH. Let also $B^{(N)}$ be the average energy rate (in energy-units per channel use) at the end of $N$ channel uses. That is,

$$
B^{(N)} \triangleq \frac{1}{N} \sum_{n=1}^{N} Y_{3, n}^{2} .
$$

The SNR of transmitter $i$ at the EH is denoted by

$$
\mathrm{SNR}_{3 i}=\frac{\left|h_{3 i}\right|^{2} P_{i}}{\sigma_{3}^{2}}
$$

Note that the maximum average energy rate $B_{\max }$ is

$B_{\max }=\sigma_{3}^{2}\left(1+\mathrm{SNR}_{31}+\mathrm{SNR}_{32}+2 \sqrt{\mathrm{SNR}_{31} \mathrm{SNR}_{32}}\right)$.

The probability of energy outage, given an average energy rate $B$, is defined as follows:

$$
P_{\text {outage }}^{(N, \epsilon)}(B) \triangleq \operatorname{Pr}\left[B^{(N)}<B-\epsilon\right],
$$

for all $B>b$ and some $\epsilon>0$. 


\section{Simultaneous Information and Energy Transmission (SIET)}

Given a minimum energy rate $b$ to be satisfied at the EH, the system is said to be operating at the information-energy rate triplet $\left(R_{1}, R_{2}, B\right) \in \mathbb{R}_{+}^{3}$ when both transmitter-receiver pairs use a transmit-receive configurations such that: (i) reliable communication at information rates $R_{1}$ and $R_{2}$ is ensured; and (ii) reliable energy transmission at energy rate $B$ is ensured. A formal definition is given below.

Definition 1 (Achievable Rates): The triplet $\left(R_{1}, R_{2}, B\right) \in$ $\mathbb{R}_{+}^{3}$ is achievable if for all $i \in\{1,2\}$, there exists a sequence of encoding functions $f_{i, 1}^{(N)}, f_{i, 2}^{(N)}, \ldots, f_{i, N}^{(N)}$ and a decoding function $\phi_{i}$ such that both the average error probability $P_{\mathrm{e}}^{(t)}(N)$, for all $t \in\{1,2, \ldots, T\}$, and the energy-outage probability $P_{\text {outage }}^{(N, \epsilon)}(B)$ tend to zero as the block-length $N$ tends to infinity. That is,

$$
\begin{gathered}
\limsup _{N \rightarrow \infty} P_{\mathrm{e}}^{(N)}=0, \text { and } \\
\limsup _{N \rightarrow \infty} P_{\text {outage }}^{(N, \epsilon)}=0 .
\end{gathered}
$$

Using Definition 1, the fundamental limits of simultaneous information and energy transmission in the Gaussian interference channel can be described by the information-energy capacity region, defined as follows.

Definition 2 (Information-Energy Capacity Region): The information-energy capacity region given a minimum energy rate $b$, denoted by $\mathcal{E}_{b}^{\mathrm{F}}$, corresponds to the closure of all achievable information-energy rate triplets $\left(R_{1}, R_{2}, B\right)$.

\section{MAIN RESULTS}

The main result consists of a description of the informationenergy capacity region $\mathcal{E}_{b}^{\mathrm{F}}$, for a given $b \geqslant 0$. Such a description is presented in the form of an approximation in the sense of the definition hereunder.

Definition 3 (Approximation of a Set): Let $n \in \mathbb{N}$ be fixed. $A$ closed and convex region $\mathcal{X} \subset \mathbb{R}_{+}^{n}$ is approximated by the sets $\underline{\mathcal{X}}$ and $\overline{\mathcal{X}}$ if $\underline{\mathcal{X}} \subseteq \mathcal{X} \subseteq \overline{\mathcal{X}}$ and $\forall \boldsymbol{x}=\left(x_{1}, \ldots, x_{n}\right) \in$ $\overline{\mathcal{X}}$ then $\left(\left(x_{1}-\xi_{1}\right)^{+},\left(x_{1}-\xi_{2}\right)^{+}, \ldots,\left(x_{n}-\xi_{n}\right)^{+}\right) \in \underline{\mathcal{X}}$, for some $\left(\xi_{1}, \xi_{2}, \ldots, \xi_{n}\right) \in \mathrm{R}_{+}^{n}$.

The following sections show that the information-energy capacity region $\mathcal{E}_{b}^{\mathrm{F}}$, with $b$ any positive real number, is approximated by the regions $\underline{\mathcal{E}}_{b}^{\mathrm{F}}$ (Theorem 1) and $\overline{\mathcal{E}}_{b}^{\mathrm{F}}$ (Theorem 2).

\section{A. Achievability}

The following theorem describes a set of rate-tuples that are achievable (Definition 1).

Theorem 1: Let $b$ be a fixed positive real. Then, the information-energy capacity region $\mathcal{E}_{b}$ contains all the rate tuples $\left(R_{1}, R_{2}, B\right)$ that satisfy for all $i \in\{1,2\}$ and $j \in$
$\{1,2\} \backslash\{i\}:$

$R_{i} \leq \frac{1}{2} \log \left(\frac{1+\left(1-\lambda_{i e}\right) \mathrm{SNR}_{i}+\left(1-\lambda_{j e}\right) \mathrm{INR}_{i}+2 \rho \sqrt{\mathrm{SNR}_{i} \mathrm{INR}_{i}}}{1+\lambda_{j p} \mathrm{INR}_{i}}\right)$

$R_{i} \leq \frac{1}{2} \log \left(\frac{1+\left(1-\left(\rho+\lambda_{i e}\right)\right) \mathrm{INR}_{j}}{1+\lambda_{i p} \mathrm{INR}_{j}}\right)$

$+\frac{1}{2} \log \left(\frac{1+\lambda_{i p} \mathrm{SNR}_{i}+\lambda_{j p} \mathrm{INR}_{i}}{1+\lambda_{j p} \mathrm{INR}_{i}}\right)$,

$R_{1}+R_{2} \leq \frac{1}{2} \log \left(\frac{1+\lambda_{i p} \mathrm{SNR}_{i}+\lambda_{j p} \mathrm{INR}_{i}}{1+\lambda_{j p} \mathrm{INR}_{i}}\right)$

$+\frac{1}{2} \log \left(\frac{1+\left(1-\lambda_{j e}\right) \mathrm{SNR}_{j}+\left(1-\lambda_{i e}\right) \mathrm{INR}_{j}+2 \rho \sqrt{\mathrm{SNR}_{j} \mathrm{INR}_{j}}}{1+\lambda_{i p} \mathrm{INR}_{j}}\right)$,

$b \leq B \leq \sigma_{3}^{2}\left(1+\mathrm{SNR}_{31}+\mathrm{SNR}_{32}+2 \rho \sqrt{\mathrm{SNR}_{31} \mathrm{SNR}_{32}}\right.$

$\left.+2 \sqrt{\lambda_{1 e} \mathrm{SNR}_{31} \lambda_{2 e} \mathrm{SNR}_{31}}\right)$,

where $\left(\rho, \lambda_{i p}, \lambda_{i e}\right) \in[0,1]^{3}$, such that $\rho+\lambda_{i p}+\lambda_{i e} \leq 1$.

Proof: The proof of Theorem 1 is presented in [14] and in the following section only a sketch of proof is described.

\section{B. Sketch of Proof of Achievability}

The achievability scheme presented in this section is built upon random coding arguments using ratesplitting [15], super-position coding, backward decoding [16], common randomness and power-spliting [12]. Let $W_{i}^{(t)} \in\left\{1,2 \ldots, 2^{N R_{i}}\right\}$ and $\Omega \in\left\{1,2 \ldots, 2^{N R_{E}}\right\}$ be the message index and the common random index at transmitter $i$ during the $t$-th block. Following a rate-splitting argument, the index $W_{i}^{(t)}$ is divided into two sub-indices $W_{i, P}^{(t)} \in\left\{1,2 \ldots, 2^{N R_{i, P}}\right\}$ and $W_{i, C}^{(t)} \in\left\{1,2 \ldots, 2^{N R_{i, C}}\right\}$, where $R_{i, C}+R_{i, P}=R_{i}$. At the end of block $t$, the message indices $W_{i, C}^{(t)}$ and $W_{i, P}^{(t)}$ must be decoded by receiver $i$, whereas $W_{i, C}^{(t)}$ must be decoded by receiver $j$, and by transmitter $j$ via feedback. Therefore at the beginning of block $t$, each transmitter possesses the knowledge of the indices $W_{1, C}^{(t-1)}$ and $W_{2, C}^{(t-1)}$. In the case of the first block $t=1$, the indices $W_{1, C}^{(0)}$ and $W_{2, C}^{(0)}$ correspond to two indices assumed to be known by all transmitters and receivers. The codebook generation at transmitter $i$ follows a four-level superposition coding scheme. The first-layer codebook is common and consists of $2^{N R_{E}}$ codewords of length $N$ symbols, denoted by $\boldsymbol{v}(1), \boldsymbol{v}(2), \ldots, \boldsymbol{v}\left(\left\lfloor 2^{N R_{E}}\right\rfloor\right)$. Note that both transmitters know $\Omega$, hence they are able to choose the same codeword $\boldsymbol{v}(\Omega)$ from the first-layer codebook. The index $\Omega$ as well as the codeword $\boldsymbol{v}(\Omega)$ are also known at the receivers, which highlights that the role of this codebook is not information transmission but energy transmission. For each codeword in the first-layer codebook, transmitters possesses a sub-codebook of $2^{N\left(R_{1, C}+R_{2, C}\right)}$ codewords. The codewords superposed on codeword $\boldsymbol{v}(\Omega)$ are denoted 
by $\quad \boldsymbol{u}(\Omega, 1,1), \boldsymbol{u}(\Omega, 1,2), \ldots, \boldsymbol{u}\left(\Omega,\left\lfloor 2^{N R_{1, C}}\right\rfloor,\left\lfloor 2^{N R_{2, C}}\right\rfloor\right)$. The resulting $\left\lfloor 2^{N R_{E}}\right\rfloor \cdot\left\lfloor 2^{N R_{1, C}}\right\rfloor \cdot\left\lfloor 2^{N R_{2, C}}\right\rfloor$ codewords are referred to as the second-layer codebook. For each codeword in the second-layer codebook there is a subcodebook of $2^{N R_{i, C}}$ codewords in the codebook of transmitter-receiver pair $i$. The codewords superposed on codeword $\boldsymbol{u}\left(\Omega, W_{1, C}^{(t-1)}, W_{2, C}^{(t-1)}\right)$ are denoted by $\boldsymbol{u}_{i}\left(\Omega, W_{1, C}^{(t-1)}, W_{2, C}^{(t-1)}, 1\right), \quad \boldsymbol{u}_{i}\left(\Omega, W_{1, C}^{(t-1)}, W_{2, C}^{(t-1)}, 2\right), \ldots$,

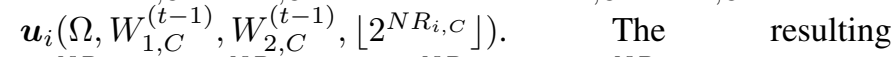
$\left\lfloor 2^{N R_{E}}\right\rfloor \cdot\left\lfloor 2^{N R_{1, C}}\right\rfloor \cdot\left\lfloor 2^{N R_{2, C}}\right\rfloor \cdot\left\lfloor 2^{N R_{i, C}}\right\rfloor$ codewords are referred to as the third-layer code. Note that this layer is particular at each transmitter-receiver pair. For each codeword in the third-layer codebook of transmitter-receiver pair $i$ there is a sub-codebook of $2^{N R_{i, P}}$ codewords. The codewords superposed on codeword $\boldsymbol{u}_{i}\left(\Omega, W_{1, C}^{(t-1)}, W_{2, C}^{(t-1)}, W_{i, C}^{(t)}\right)$ are denoted by $\boldsymbol{s}_{i}\left(\Omega, W_{1, C}^{(t-1)}, W_{2, C}^{(t-1)}, W_{i, C}^{(t)}, 1\right)$, $\boldsymbol{s}_{i}\left(\Omega, W_{1, C}^{(t-1)}, W_{2, C}^{(t-1)}, W_{i, C}^{(t)}, 2\right), \ldots$

$\boldsymbol{s}_{i}\left(\Omega, W_{1, C}^{(t-1)}, W_{2, C}^{(t-1)}, W_{i, C}^{(t)},\left\lfloor 2^{\left.\left.N R_{i, P}\right\rfloor\right)}\right.\right.$. The resulting

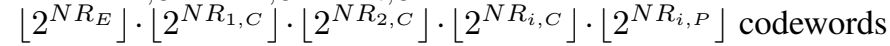
are referred to as the four layer code and as the previous code layer, this layer is particular to each transmitter-receiver pair. For transmitting the triplet $\left(\Omega, W_{1, C}^{(t-1)}, W_{2, C}^{(t-1)}, W_{i, C}^{(t)}, W_{i, P}^{(t)}\right)$ from transmitter $i$, the channel input-symbol $X_{i, n}$ at channel use $n \in\{1,2, \ldots, N\}$ is a deterministic function of the $n$-th components of the codewords $v(\Omega)$, $\boldsymbol{u}\left(\Omega, W_{1, C}^{(t-1)}, W_{2, C}^{(t-1)}\right), \quad \boldsymbol{u}_{i}\left(\Omega, W_{1, C}^{(t-1)}, W_{2, C}^{(t-1)}, W_{i, C}^{(t)}\right) \quad$ and $\boldsymbol{s}_{i}\left(\Omega, W_{1, C}^{(t-1)}, W_{2, C}^{(t-1)}, W_{i, C}^{(t)}, W_{i, P}^{(t)}\right)$. The information rates $\left(R_{1}, R_{2}\right)$ that are achievable by the code described above satisfy the following inequalities.

Lemma 1: The set of achievable information rate pairs $\left(R_{1}, R_{2}\right)$ satisfies the following inequalities for all $i \in\{1,2\}$ and $j \in\{1,2\} \backslash\{i\}$ :

$$
\begin{aligned}
R_{i} \leq & I\left(U, X_{i}, U_{j} ; Y_{i} \mid V\right), \\
R_{i} \leq & I\left(U_{i} ; Y_{j} \mid U, X_{j}, V\right) \\
& +I\left(X_{i} ; Y_{i} \mid U_{i}, U_{j}, U, V\right), \\
R_{1}+R_{2} \leq & I\left(X_{i} ; Y_{i} \mid U_{i}, U_{j}, U, V\right) \\
& +I\left(V, U_{j}, X_{i} ; Y_{i}\right),
\end{aligned}
$$

for a given joint distribution $P_{V U U_{1} U_{2} S_{1} S_{2}}\left(v, u, u_{1}, u_{2}, s_{1}, s_{2}\right)$ $\begin{array}{lllll}\text { that factorizes as } & P_{V}(v) & P_{U \mid V}(u \mid v) & P_{U_{1} \mid U V}\left(u_{1} \mid u v\right)\end{array}$ $P_{U_{2} \mid U V}\left(u_{2} \mid u v\right) \quad P_{S_{1} \mid U U_{1} V}\left(s_{1} \mid u u_{1} v\right) \quad P_{S_{2} \mid U U_{2} V}\left(s_{2} \mid u u_{2} v\right)$ and $X_{i}=\theta_{i}\left(V, U, U_{i}, S_{i}\right)$, with $\theta_{1}$ and $\theta_{2}$ injective functions.

Proof: The proof of Lemma 1 uses standard random coding arguments and is presented in [14].

For all $k \in\{1,2\}$ and a fixed tuple $\left(\rho, \lambda_{k c}, \lambda_{k p}, \lambda_{k e}\right) \in[0,1]^{4}$ such that $\rho+\lambda_{k c}+\lambda_{k p}+\lambda_{k e}=1$, consider the following random variables: $V \sim \mathcal{N}(0,1) ; U \sim \mathcal{N}(0, \rho) ; U_{k} \sim \mathcal{N}\left(0, \lambda_{k c}\right)$; and $S_{k} \sim \mathcal{N}\left(0, \lambda_{k p}\right)$, which are independent of each other. Let the channel input of transmitter $k$ be

$$
X_{k}=\sqrt{P_{k}} U+\sqrt{P_{k}} S_{k}+\sqrt{P_{k}} U_{k}+\sqrt{\lambda_{k e} P_{k}} V
$$

The choice of this input distribution yields

$$
\begin{aligned}
& I\left(U, X_{i}, U_{j} ; Y_{i} \mid V\right)= \\
& \frac{1}{2} \log \left(\frac{1+\left(1-\lambda_{i e}\right) \mathrm{SNR}_{i}+\left(1-\lambda_{j e}\right) \mathrm{INR}_{i}+2 \rho \sqrt{\mathrm{SNR}_{i} \mathrm{INR}_{i}}}{1+\lambda_{j p} \mathrm{INR}_{i}}\right),
\end{aligned}
$$

$$
\begin{aligned}
& I\left(U_{i}, X_{i}, U_{j} ; Y_{i} \mid V\right)=\frac{1}{2} \log \left(\frac{1+\left(1-\left(\rho+\lambda_{i e}\right)\right) \mathrm{INR}_{j}}{1+\lambda_{i p} \mathrm{INR}_{j}}\right),(20 \mathrm{~b}) \\
& I\left(X_{i} ; Y_{i} \mid U, U_{i}, U_{j}, V\right)=\frac{1}{2} \log \left(\frac{1+\lambda_{i p} \mathrm{SNR}_{i}+\lambda_{j p} \mathrm{INR}_{i}}{1+\lambda_{j p} \mathrm{INR}_{i}}\right) .
\end{aligned}
$$

Finally, plugging (20) into (18) completes the proof of (17a) - (17c).

The average received energy rate $\bar{B}$ achieved by using the code described above is given by

$$
\begin{aligned}
\bar{B}= & E\left[Y_{3, n}^{2}\right] \\
= & h_{31}^{2} E\left[X_{1, n}^{2}\right]+h_{32}^{2} E\left[X_{2, n}^{2}\right]+2 h_{31} h_{32} E\left[X_{1, n} X_{2, n}\right]+\sigma_{3}^{2} \\
\leq & h_{31}^{2} P_{1}+h_{32}^{2} P_{2}+2\left|h_{31}\right|\left|h_{32}\right| \sqrt{P_{1} P_{2}}\left(\rho+\sqrt{\lambda_{1 e} \lambda_{2 e}}\right)+\sigma_{3}^{2} \\
= & \sigma_{3}^{2}\left(1+\operatorname{SNR}_{31}+\operatorname{SNR}_{32}\right. \\
& \left.+2 \sqrt{\operatorname{SNR}_{31} \operatorname{SNR}_{32}}\left(\rho+\sqrt{\lambda_{1 e} \lambda_{2 e}}\right)\right)
\end{aligned}
$$

From the weak law of large numbers [17], it holds that $\forall \epsilon>0$,

$$
\lim _{N \rightarrow \infty} \operatorname{Pr}\left(B^{(N)}<\bar{B}-\epsilon\right)=0
$$

From (21), it holds that for any energy rate $B$ that satisfies $0<B \leq \bar{B}$, it holds that

$$
\lim _{N \rightarrow \infty} \operatorname{Pr}\left(B^{(N)}<B-\epsilon\right)=0
$$

which proves (17d) and completes the sketch of proof.

\section{Converse}

The following theorem describes a converse region denoted by $\overline{\mathcal{E}}_{b}^{\mathrm{F}}$.

Theorem 2: Let $b$ be a fixed positive real. Then, the information-energy capacity region $\mathcal{E}_{b}^{\mathrm{F}}$ is contained into the set of all the rate tuples $\left(R_{1}, R_{2}, B\right)$ that satisfy for all $i \in\{1,2\}$ 
and $j \in\{1,2\} \backslash\{i\}$ :

$$
\begin{aligned}
R_{i} \leq & \frac{1}{2} \log \left(1+\beta_{i} \mathrm{SNR}_{i}+\beta_{j} \mathrm{INR}_{i}+2 \rho \sqrt{\beta_{i} \mathrm{SNR}_{i} \beta_{j} \mathrm{INR}_{i}}\right), \\
R_{i} \leq & \frac{1}{2} \log \left(1+\frac{\left.\beta_{i}\left(1-\rho^{2}\right)\right) \mathrm{SNR}_{i}}{1+\beta_{i}\left(1-\rho^{2}\right) \mathrm{INR}_{j}}\right) \\
& +\frac{1}{2} \log \left(1+\beta_{i}\left(1-\rho^{2}\right) \mathrm{INR}_{j}\right), \\
R_{1}+R_{2} \leq & \frac{1}{2} \log \left(1+\frac{\left.\beta_{i}\left(1-\rho^{2}\right)\right) \mathrm{SNR}_{i}}{1+\beta_{i}\left(1-\rho^{2}\right) \mathrm{INR}_{j}}\right) \\
+ & \frac{1}{2} \log \left(1+\beta_{j} \mathrm{SNR}_{j}+\beta_{i} \mathrm{INR}_{j}+2 \rho \sqrt{\left.\beta_{j} \mathrm{SNR}_{j} \beta_{i} \mathrm{INR}_{j}\right),}\right. \\
B \leq & \sigma_{3}^{2}\left(1+\mathrm{SNR}_{31}+\mathrm{SNR}_{32}+2 \rho \sqrt{\beta_{1} \mathrm{SNR}_{31} \beta_{2} \mathrm{SNR}_{32}}\right. \\
& \left.+2 \sqrt{\left(1-\beta_{1}\right) \mathrm{SNR}_{31}\left(1-\beta_{2}\right) \mathrm{SNR}_{32}}\right),
\end{aligned}
$$

with $\left(\beta_{1}, \beta_{2}\right) \in[0,1]^{2}$.

Proof: The proof of Theorem 2 is presented in [14] and in the following section only a sketch of proof is described.

\section{Sketch of Proof of the Converse}

Fix an information-energy rate triplet $\left(R_{1}, R_{2}, B\right)$ achievable with a given coding scheme (Definition 1 ). Denote by $\boldsymbol{X}_{1}$ and $\boldsymbol{X}_{2}$ the channel inputs resulting from transmitting the independent messages $\left(W_{1}, \Omega\right)$ and $\left(W_{2}, \Omega\right)$ using such coding scheme. Denote by $\boldsymbol{Y}_{1}$ and $\boldsymbol{Y}_{2}$ the corresponding channel outputs. Define the following random variables:

$$
\begin{aligned}
& S_{1}=h_{21} X_{1}+Z_{2}, \text { and } \\
& S_{2}=h_{12} X_{2}+Z_{1},
\end{aligned}
$$

where, $Z_{1}$ and $Z_{2}$ are real Gaussian random variables independent of each other with zero means and variances $\sigma_{1}^{2}$ and $\sigma_{2}^{2}$, respectively. Using assumption (15) and Fano's inequality and following similar steps as in [16], it can be shown that the information rates $R_{1}$ and $R_{2}$ must satisfy the following inequalities for all $i \in\{1,2\}$ and $j \in\{1,2\} \backslash\{i\}$ :

$$
\begin{aligned}
N R_{i} \leq & \sum_{n=1}^{N}\left[h\left(Y_{i, n}\right)-h\left(Z_{i, n}\right)\right]+\mathrm{o}(N) \\
N R_{i} \leq & \sum_{n=1}^{N}\left[h\left(Y_{j, n}\right) \mid X_{j, n}\right)-h\left(Z_{j, n}\right) \\
& \left.+h\left(Y_{i, n} \mid X_{j, n}, S_{i, n}\right)-h\left(Z_{i, n}\right)\right]+\mathrm{o}(N), \\
N\left(R_{1}+R_{2}\right) \leq & \sum_{n=1}^{N}\left[h\left(Y_{i, n} \mid S_{i, n}, X_{j, n}\right)\right. \\
& \left.-h\left(Z_{i, n}\right)+h\left(Y_{j, n}\right)-h\left(Z_{j, n}\right)\right]+\mathrm{o}(N),
\end{aligned}
$$

where $\frac{\mathrm{o}(N)}{N}$ tends to zero as $N$ tends to infinity.
Using assumption (16), for a given $\epsilon_{N}>0$ and an $\eta>0$, there exist $N_{0}(\eta)$ such that for any $N \geq N_{0}(\eta)$ it holds that

$$
\operatorname{Pr}\left(B^{(N)}<B-\epsilon_{N}\right)<\eta \text {. }
$$

Equivalently,

$$
\operatorname{Pr}\left(B^{(N)} \geq B-\epsilon_{N}\right) \geq 1-\eta .
$$

From Markov's inequality, the following holds:

$$
\left(B-\epsilon_{N}\right) \operatorname{Pr}\left(B^{(N)} \leq B-\epsilon_{N}\right) \leq E\left[B^{(N)}\right] .
$$

Combining (26) and (27) yields

$$
\left(B-\epsilon_{N}\right)(1-\eta) \leq E\left[B^{(N)}\right],
$$

which can be written as

$$
\left(B-\delta_{N}\right) \leq E\left[B^{(N)}\right],
$$

for some $\delta_{N}>\epsilon_{N}$ and a sufficiently large $N$.

In the following, for all $n \in \mathbb{N}$, the bounds in (24) and (29) are evaluated assuming that the channel inputs $X_{1, n}$ and $X_{2, n}$ are arbitrary correlated random variables with

$$
\begin{aligned}
\mu_{i, n} & \triangleq \mathrm{E}\left[X_{i, n}\right], \\
\gamma_{i, n}^{2} & \triangleq \operatorname{Var}\left[X_{i, n}\right], \\
\lambda_{n} & \triangleq \operatorname{Cov}\left[X_{1, n} X_{2, n}\right] .
\end{aligned}
$$

The input sequences must satisfy the input power constraint (4) which can be written for $i \in\{1,2\}$, as

$$
\frac{1}{N} \sum_{n=1}^{N} \mathrm{E}\left[X_{i, n}^{2}\right]=\left(\frac{1}{N} \sum_{n=1}^{N} \gamma_{i, n}^{2}\right)+\left(\frac{1}{N} \sum_{n=1}^{N} \mu_{i, n}^{2}\right) \leqslant P_{i} .
$$

Using these elements, the terms in the right-hand side of (24) can be upper-bounded as follows:

$h\left(Y_{i, n}\right) \leqslant \frac{1}{2} \log _{2}\left(2 \pi e\left(h_{i i}^{2} \gamma_{i, n}^{2}+h_{i j}^{2} \gamma_{j, n}^{2}+2 h_{i i} h_{i j} \lambda_{n}+\sigma_{1}^{2}\right)\right)$,

$h\left(Y_{j, n} \mid X_{j, n}\right) \leqslant \frac{1}{2} \log \left(1+\gamma_{i, n}^{2}\left(1-\frac{\lambda_{n}^{2}}{\gamma_{i, n}^{2} \gamma_{j, n}^{2}}\right) \frac{h_{j i}^{2}}{\sigma_{j}^{2}}\right)$

$+\frac{1}{2} \log \left(2 \pi e \sigma_{j}^{2}\right)$, and

$h\left(Y_{i, n} \mid X_{j, n}, S_{i, n}\right) \leqslant \frac{1}{2} \log \left(1+\frac{\gamma_{i, n}^{2}\left(1-\frac{\lambda_{n}^{2}}{\gamma_{i, n}^{2} \gamma_{j, n}^{2}}\right) \frac{h_{i i}^{2}}{\sigma_{i}^{2}}}{1+\gamma_{i, n}^{2}\left(1-\frac{\lambda_{n}^{2}}{\gamma_{i, n}^{2} \gamma_{j, n}^{2}}\right) \frac{h_{j i}^{2}}{\sigma_{j}^{2}}}\right)$ $+\frac{1}{2} \log \left(2 \pi e \sigma_{i}^{2}\right)$

The expectation of the average received energy rate is given by

$$
\begin{aligned}
& \mathrm{E}\left[B^{(N)}\right]=\mathrm{E}\left[\frac{1}{N} \sum_{n=1}^{N} Y_{3, n}^{2}\right]= \\
& h_{31}^{2}\left(\frac{1}{N} \sum_{n=1}^{N}\left(\gamma_{1, n}^{2}+\mu_{1, n}^{2}\right)\right)+h_{32}^{2}\left(\frac{1}{N} \sum_{n=1}^{N}\left(\gamma_{2, n}^{2}+\mu_{2, n}^{2}\right)\right) \\
& +2 h_{31} h_{32}\left(\frac{1}{N} \sum_{n=1}^{N}\left(\lambda_{n}+\mu_{1, n} \mu_{2, n}\right)\right)+\sigma_{3}^{2} .
\end{aligned}
$$


Using Cauchy-Schwarz inequality, the energy rate in (35) can be upper-bounded as follows:

$\mathrm{E}\left[B^{(N)}\right] \leqslant \sigma_{3}^{2}+h_{31}^{2}\left(\frac{1}{N} \sum_{n=1}^{N}\left(\gamma_{1, n}^{2}+\mu_{1, n}^{2}\right)\right)$

$+h_{32}^{2}\left(\frac{1}{N} \sum_{n=1}^{N}\left(\gamma_{2, n}^{2}+\mu_{2, n}^{2}\right)\right)$

$2 h_{31} h_{32}\left(\left|\frac{1}{N} \sum_{n=1}^{N} \lambda_{n}\right|+\left(\frac{1}{N} \sum_{n=1}^{N} \mu_{1, n}^{2}\right)^{1 / 2}\left(\frac{1}{N} \sum_{n=1}^{N} \mu_{2, n}^{2}\right)^{1 / 2}\right)$.

Combining (29) and (36) yields the following upper-bound on the energy rate $B$ :

$B \leqslant \sigma_{3}^{2}+\frac{h_{31}^{2}}{N} \sum_{n=1}^{N}\left(\gamma_{1, n}^{2}+\mu_{1, n}^{2}\right)+\frac{h_{32}^{2}}{N} \sum_{n=1}^{N}\left(\gamma_{2, n}^{2}+\mu_{2, n}^{2}\right)$

$2 h_{31} h_{32}\left(\left|\frac{1}{N} \sum_{n=1}^{N} \lambda_{n}\right|+\left(\frac{1}{N} \sum_{n=1}^{N} \mu_{1, n}^{2}\right)^{1 / 2}\left(\frac{1}{N} \sum_{n=1}^{N} \mu_{2, n}^{2}\right)^{1 / 2}\right)$ $+\delta_{N}$.

Consider the following definitions, for all $i \in\{1,2\}$ :

$$
\begin{aligned}
\mu_{i}^{2} & \triangleq \frac{1}{N} \sum_{n=1}^{N} \mu_{i, n}^{2}, \\
\gamma_{i}^{2} & \triangleq \frac{1}{N} \sum_{n=1}^{N} \gamma_{i, n}^{2}, \text { and } \\
\beta_{i} & \triangleq \frac{\gamma_{i}^{2}}{P_{i}} \\
\rho & \triangleq \frac{\left(\frac{1}{N} \sum_{n=1}^{N} \lambda_{n}\right)}{\left|\gamma_{1}\right|\left|\gamma_{2}\right|}
\end{aligned}
$$

Plugging (34) in (24) and after some manipulations using the definitions in (38) yields:

$$
\begin{aligned}
& R_{i} \leqslant \frac{1}{2} \log \left(\frac{h_{i i}^{2} \gamma_{i}^{2}}{\sigma_{i}^{2}}+\frac{h_{i j}^{2} \gamma_{j}^{2}}{\sigma_{i}^{2}}+2 \rho \sqrt{\frac{h_{i i}^{2} h_{i j}^{2} \gamma_{i}^{2} \gamma_{j}^{2}}{\sigma_{i}^{4}}}\right),(3 \\
& R_{i} \leqslant \frac{1}{2} \log \left(1+\frac{h_{j i}^{2}}{\sigma_{j}^{2}} \gamma_{i}^{2}\left(1-\rho^{2}\right)\right) \\
& +\frac{1}{2} \log \left(1+\frac{\gamma_{i}^{2}\left(1-\rho^{2}\right) \frac{h_{i i}^{2}}{\sigma_{i}^{2}}}{1+\gamma_{i}^{2}\left(1-\rho^{2}\right) \frac{h_{j i}^{2}}{\sigma_{j}^{2}}}\right) \\
& R_{1}+R_{2} \leqslant \frac{1}{2} \log \left(\frac{h_{j j}^{2} \gamma_{j}^{2}}{\sigma_{j}^{2}}+\frac{h_{j i}^{2} \gamma_{i}^{2}}{\sigma_{j}^{2}}+2 \rho \sqrt{\frac{h_{j j}^{2} h_{j i}^{2} \gamma_{i}^{2} \gamma_{j}^{2}}{\sigma_{j}^{4}}}\right)
\end{aligned}
$$

and for all $\left(R_{1}, R_{2}, B\right) \in \overline{\mathcal{E}}_{b}^{\mathrm{F}}$ it follows that $\left(\left(R_{1}-1\right)^{+},\left(R_{2}-\right.\right.$ $\left.1)^{+},\left(B-\frac{B_{\max }}{2}\right)^{+}\right) \in \underline{\mathcal{E}}_{b}^{\mathrm{F}}$.

Proof: Following similar steps as in [16], it can be shown that for all $\left(R_{1}, R_{2}, 0\right) \in \overline{\mathcal{E}}_{b}^{\mathrm{F}}$ it follows that $\left(\left(R_{1}-1\right)^{+},\left(R_{2}-\right.\right.$ $\left.1)^{+}, 0\right) \in \underline{\mathcal{E}}_{b}^{\mathrm{F}}$. Note also that for all $\left(R_{1}, R_{2}, B\right) \in \overline{\mathcal{E}}_{b}^{\mathrm{F}}$ and for all $\left(R_{1}, R_{2}, B^{\prime}\right) \in \underline{\mathcal{E}}_{b}^{\mathrm{F}}$, there always exists a tuple $\left(\beta_{1}, \beta_{2}, \lambda_{1 e}, \lambda_{2 e}, \rho\right)$ such that:

$$
\begin{aligned}
& \frac{B-B^{\prime}}{B_{\max }} \\
& \leq \frac{2\left|h_{31}\right|\left|h_{32}\right| \sqrt{P_{1} P_{2}}\left(\sqrt{\left(1-\beta_{1}\right)\left(1-\beta_{2}\right)}+\rho \sqrt{\beta_{1} \beta_{2}}\right)}{\sigma_{3}^{2}+h_{31}^{2} P_{1}+h_{32}^{2} P_{2}+2\left|h_{31}\right|\left|h_{32}\right| \sqrt{P_{1} P_{2}}} \\
& \leq \frac{2 \sqrt{\mathrm{SNR}_{31} \mathrm{SNR}_{32}}}{1+\mathrm{SNR}_{31}+\mathrm{SNR}_{32}+2 \sqrt{\mathrm{SNR}_{31} \mathrm{SNR}_{32}}} \\
& \leq \frac{2 \sqrt{\mathrm{SNR}_{31} \mathrm{SNR}_{32}}}{1+4 \sqrt{\mathrm{SNR}_{31} \mathrm{SNR}_{32}}} \leq \frac{1}{2},
\end{aligned}
$$

which completes the proof.

\section{EXAMPLE}

Consider a Gaussian interference channel with feedback and an external EH with parameters $\mathrm{SNR}_{1}=\mathrm{SNR}_{2}=20 \mathrm{~dB}$, $\mathrm{INR}_{1}=\mathrm{INR}_{2}=\mathrm{SNR}_{31}=\mathrm{SNR}_{32}=10 \mathrm{~dB}$ and $\sigma_{3}^{2}=1$. Figure 2 and Figure 3 show $\underline{\mathcal{E}}_{b}$ and $\overline{\mathcal{E}}_{b}$, respectively, with $b=0$. Figure 4 shows both $\underline{\mathcal{E}}_{b}$ and $\overline{\mathcal{E}}_{b}$ in the same axes. Note that for all $B \in\left[0,1+\mathrm{SNR}_{31}+\mathrm{SNR}_{32}\right]$, transmitting information with independent codewords is enough to satisfy the energy rate constraints. This implies that $\beta_{1}=\beta_{2}=1$ is optimal in this regime. Alternatively, for all $B \in\left[1+\mathrm{SNR}_{31}+\right.$ $\left.\mathrm{SNR}_{32}, B_{\max }\right]$, transmitters deal with a trade-off between the information and energy rate. More specifically, increasing $B$ reduces the information region and makes the informationenergy capacity region shrink. 


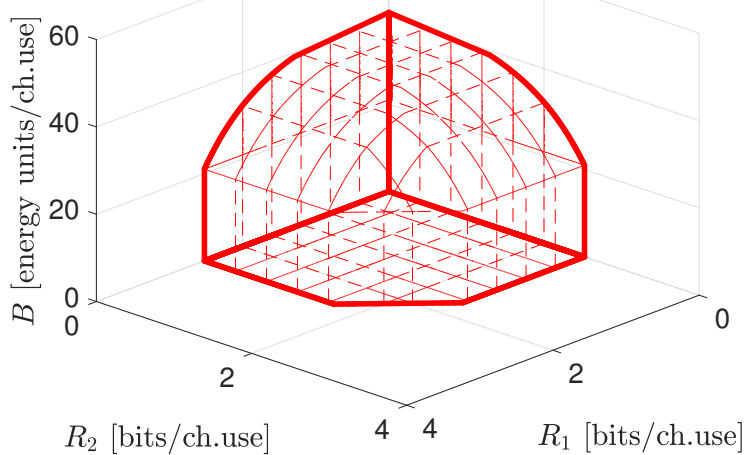

Fig. 2. 3-D representation of $\underline{\mathcal{E}}_{b}^{\mathrm{F}}$, with parameters $\mathrm{SNR}_{1}=\mathrm{SNR}_{2}=20$ $\mathrm{dB}, \mathrm{INR}_{1}=\mathrm{INR}_{2}=\mathrm{SNR}_{31}=\mathrm{SNR}_{32}=10 \mathrm{~dB}$ and $\sigma_{3}^{2}=1$.

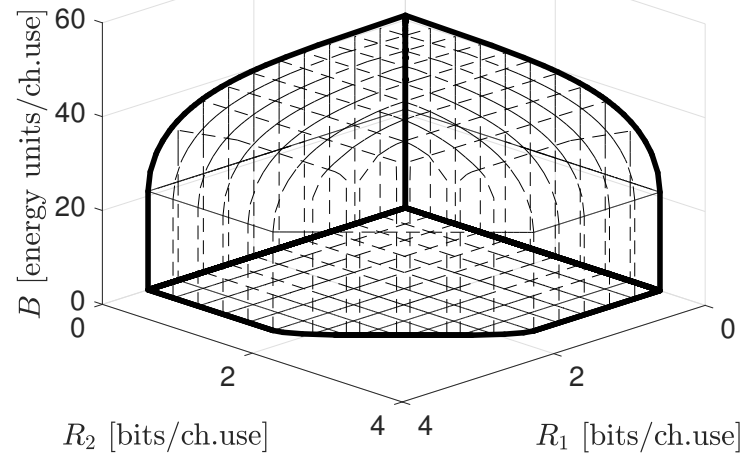

Fig. 3. 3-D representation of $\overline{\mathcal{E}}_{b}^{\mathrm{F}}$, with parameters $\mathrm{SNR}_{1}=\mathrm{SNR}_{2}=20$ $\mathrm{dB}, \mathrm{INR}_{1}=\mathrm{INR}_{2}=\mathrm{SNR}_{31}=\mathrm{SNR}_{32}=10 \mathrm{~dB}$ and $\sigma_{3}^{2}=1$.

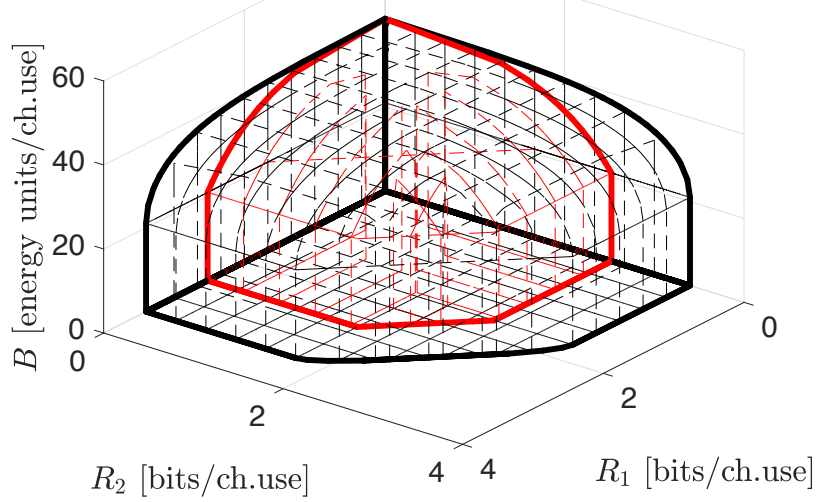

Fig. 4. 3-D superposition of $\mathcal{E}_{b}^{\mathrm{F}}$ and $\overline{\mathcal{E}}_{b}^{\mathrm{F}}$, with parameters $\mathrm{SNR}_{1}=\mathrm{SNR}_{2}=$ $20 \mathrm{~dB}, \mathrm{INR}_{1}=\mathrm{INR}_{2}=\mathrm{SNR}_{31}=\mathrm{SNR}_{32}=10 \mathrm{~dB}$ and $\sigma_{3}^{2}=1$.

\section{CONCLUSIONS}

The fundamental limits of simultaneous information and energy transmission in the two-user Gaussian interference channel with perfect output feedback have been approximated. This approximation consists of an achievable region and a converse region. The achievable region is obtained using random coding arguments and techniques that are already known, e.g., rate splitting, common randomness, superposition coding, power-splitting and block Markov decoding. The novelty of this achievability scheme lies in the superposition of the information-carrying layers (second, third and fourth layers) over the energy-carrying code-layer (first layer). The converse region is obtained using Fano's inequality, cut-set bounds and genie aided scenarios to obtain the bounds on the information rates. As for the upper-bound on the energy rate, the ingredients are simply the weak law of large numbers and Chebyshev's inequality.

\section{REFERENCES}

[1] S. Ulukus, A. Yener, E. Erkip, O. Simeone, M. Zorzi, P. Grover, and K. Huang, "Energy harvesting wireless communications: A review of recent advances," IEEE Journal on Selected Areas in Communications, vol. 33, no. 3, pp. 360-381, Mar. 2015.

[2] K. Tutuncuoglu and A. Yener, "Sum-rate optimal power policies for energy harvesting transmitters in an interference channel," J. Commun. and Netw., vol. 14, no. 2, pp. 151-161, Apr. 2012.

[3] S. Chao, L. Wei-Chiang, and C. Tsung-Hui, "Simultaneous information and energy transfer: A two-user MISO interference channel case," in Proc. IEEE Global Communications Conference, Anaheim, CA, USA, Dec. 2012, pp. 3862-3867.

[4] K. Tutuncuoglu and A. Yener, "Optimum transmission policies for battery limited energy harvesting nodes," IEEE Trans. Wireless Commun., vol. 11, no. 3, pp. 1180-1189, Mar. 2012.

[5] J. Park and B. Clerckx, "Joint wireless information and energy transfer in a two-user MIMO interference channel," IEEE Trans. Wireless Commun., vol. 12, no. 8, pp. 4210-4221, Aug. 2013.

[6] B. Calise, Y. Zhang, and M. Amin, "Simultaneous transfer of energy and information for MIMO-OFDM relay system," in Proc. IEEE International Conference on Communications in China, Beijing, China, Aug. 2012.

[7] N. Tesla, "Apparatus for transmitting electrical energy." Dec. 1 1914, US Patent 1,119,732.

[8] L. R. Varshney, "Transporting information and energy simultaneously," in Proc. IEEE International Symposium on Information Theory, Toronto, ON, Canada, Jul. 2008, pp. 1612-1616.

[9] - "On energy/information cross-layer architectures," in Proc. IEEE International Symposium on Information Theory, Cambridge, MA, USA, Jul. 2012, pp. 1356-1360.

[10] P. Grover and A. Sahai, "Shannon meets Tesla: Wireless information and power transfer," in Proc. IEEE International Symposium on Information Theory, Austin, TX, USA, Jun. 2010, pp. 2363-2367.

[11] A. M. Fouladgar; and O. Simeone, "On the transfer of information and energy in multi-user systems," IEEE Communications Letters, vol. 16, no. 11, pp. 1733-1736, Nov. 2012.

[12] S. Belhadj Amor, S. M. Perlaza, I. Krikidis, and H. V. Poor, "Feedback enhances simultaneous wireless information and energy transmission in multiple access channels," IEEE Trans. Inf. Theory, vol. 63, no. 8, pp. 5244-5265, Aug. 2017.

[13] S. Belhadj Amor and S. M. Perlaza, "Fundamental limits of simultaneous energy and information transmission," in Proc. International Symposium on Telecommunications, Thessaloniki, Greece, May 2016.

[14] N. Khalfet and S. M. Perlaza, "Simultaneous information and energy transmission in the interference channel," INRIA Grenoble - RhôneAlpes, Tech. Rep. 9102, Oct. 2017.

[15] T. S. Han and K. Kobayashi, "A new achievable rate region for the interference channel," IEEE Trans. Inf. Theory, vol. 27, pp. 49-60, Jan. 1981.

[16] C. Suh and D. N. C. Tse, "Feedback capacity of the Gaussian interference channel to within 2 bits," IEEE Trans. Inf. Theory, vol. 57, no. 5, pp. 2667-2685, May 2011.

[17] R. Ash, Information Theory. New York, USA: Dover publications, Inc, 1990. 